\title{
Transthyretin Amyloidosis with Gastrointestinal Manifestation: a Case Report
}

\author{
Radislav Nakov ${ }^{1,2}$, Stayko Sarafov ${ }^{3}$, Ventsislav Nakov ${ }^{1,2}$, Mariana Gospodinova, ${ }^{2,4}$, Tihomir Todorov ${ }^{5}$ Andrey Kirov ${ }^{2,5}$, \\ Albena Todorova ${ }^{2,5}$, Ivailo Tournev ${ }^{2,3,6}$
}

1) Tsaritsa Yoanna University Hospital, Sofia

2) Medical University of Sofia 3) Clinic of Nervous Diseases, Alexandrovska University Hospital, Sofia

4) Clinic of Cardiology,

Medical Institute of Ministry of Interior, Sofia

5) Genetic and Medicodiagnostic Laboratory

“Genica”, Sofia

6) Department of Cognitive Science and Psychology, New Bulgarian University, Sofia Bulgaria

\begin{abstract}
Transthyretin amyloidosis (ATTR) is a rare, progressive, life-threatening, hereditary disorder caused by mutations in the transthyretin gene. Due to the phenotypic heterogeneity, ATTR is difficult to recognize and it is often diagnosed very late. In ATTR gastrointestinal (GI) disorders play an important role in the patients' morbidity and mortality. In some cases, GI symptoms are present even before the onset of the peripheral polyneuropathy. However, the complaints are various and it is really difficult to differentiate them from other GI disorders. We present a 61-year old male referred for diarrhea, unintentional weight loss and early satiety. He had hypotension after longstanding hypertension, numbness and tingling in the feet. We considered a broad differential diagnosis spectrum of chronic diarrhea syndrome and performed numerous laboratory, biochemical, imaging, endoscopic, histological and genetic tests. Transthyretin amyloidosis with a Glu89Gln mutation was diagnosed. Transthyretin amyloidosis is frequently misdiagnosed, representing a diagnostic challenge in GI practice. The presence of certain clinical combinations could help gastroenterologists to include ATTR in their diagnostic work-up.
\end{abstract}

Key words: transthyretin - amyloidosis - chronic diarrhea - misdiagnoses.

Abbreviations: ATTR: transthyretin amyloidosis; GI: gastrointestinal; NCWS: non-celiac wheat sensitivity; TTR: transthyretin.

\section{INTRODUCTION}

Transthyretin amyloidosis (ATTR) is a rare, systemic, hereditary autosomal dominant disease caused by mutations in the gene encoding the synthesis of the transport protein transthyretin (TTR) $[1,2]$. The TTR is a serum protein that transports thyroxine and retinol [vitamin A] into the plasma [2]. It is also found in the cerebrospinal fluid and it is synthesized mainly by the liver and choroid plexus in the central nervous system. The TTR gene is located on the long arm of chromosome 18 [3].

The advanced age and amyloidogenic TTR mutations lead to a decreased stability of the TTR tetramer, making it more susceptible to dissociation of monomers different in structure from the genuine ones. Monomers aggregate into beta structured fibrils that form extracellular amyloid deposits and lead to degenerative changes and impaired function of many organs [4]. Due to the phenotypic heterogeneity, ATTR is difficult to recognize and it is often diagnosed very late. The most common ATTR mutations are Val30Met, Glu89Gln, Ser77Phe and Ser52Pro [5].

\section{CASE REPORT}

A 61-year old male was referred to the Clinic of Gastroenterology, "Tsaritsa Yoanna” University Hospital, complaining of about eight watery stools per day, mainly postprandial and during the night, a weight loss of approximately $20 \mathrm{~kg}$ for the last two years, early satiety and presence of undigested food particles in the stool. Furthermore, the patient had tingling and numbness in the hands and feet and a recent onset of low blood pressure, although he had been known to have hypertension for years. A history of more than 20 collapses over the past two years was reported, which were considered as symptoms of epilepsy. One year ago, when diarrhea started, the patient received an endoscopy and a tubular polyp from the descending colon had been removed. At the present 
admission, all laboratory and biochemical results were within the reference range.

We started a diagnostic work-up of the chronic diarrhea syndrome. The microbiological cultures ruled out parasites or enteropathogenic bacteria, including Clostridium difficile. Fecal calprotectin was $99 \mu \mathrm{g} / \mathrm{g}$, demonstrating mild intestinal inflammation. Thyroid pathology was also excluded. Chromogranin A was normal, excluding a neuroendocrine tumor. Hydrogen breath test with lactulose excluded smallintestinal bacterial overgrowth but showed a shortened orocecal transit time (30 minutes). Faecal pancreatic elastase $(139 \mu \mathrm{g} / \mathrm{g})$ suggested moderate pancreatic exocrine insufficiency; however, the serum amylase and lipase were within the reference range and the abdominal ultrasonography and computer tomography scan with contrast did not show lesions in the pancreas. Celiac disease was discussed as a possible diagnosis in this patient due to the presence of a typical sonographic pattern - dilated small bowel loops with increased fluid content and rapid peristalsis. However, serology and duodenal histology were negative. Moreover, PAS-positive macrophages were not detected in the duodenal biopsies, thus excluding Whipple's disease. Ileocolonoscopy with staging biopsies excluded ulcerative colitis, Crohn's disease, microscopic colitis, and neoplastic disease. We recommended videocapsule endoscopy, but the patient refused, because the National Healthcare Insurance Fund does not cover this procedure.

In summary, we found a moderate exocrine pancreatic insufficiency and shortened oro-cecal transit time that could not explain all the complaints of our patient. We commenced empirical antibiotic therapy with Ciprofloxacin 500mg BID and Metronidazole 500mg TID and also prescribed pancreatic enzymes $95000 \mathrm{IU}$ and Saccharomyces boulardii.

One month later the patient presented in our outpatient clinic with no significant improvement: five watery stools per day and anorexia, but with normal faecal elastase $(>200$ $\mu \mathrm{g} / \mathrm{g})$ and faecal calprotectin $(45 \mu \mathrm{g} / \mathrm{g})$. He had still low blood pressure and syncopes; therefore, we referred him to a cardiologist who performed echocardiography that described a significant left and right ventricular hypertrophy, systolic and diastolic dysfunction, thickened interatrial septum, suggesting infiltrative cardiomyopathy. A rectal biopsy was recommended to exclude amyloidosis. The patient refused a second total colonoscopy, so we performed a rectoscopy. In all the three rectal biopsies stained with Congo Red, no amyloid inclusions were detected.

After a multidisciplinary team discussion, we decided that the patient might have non-celiac wheat sensitivity (NCWS), which is defined by the presence of GI and extra-intestinal symptoms following the ingestion of gluten-containing cereals in subjects without celiac disease or wheat allergy [6]. Our patient answered this definition, so we started a gluten-free diet. Significant amelioration of symptoms was observed during the first three weeks, followed by a dramatic deterioration.

This raised new questions about the diagnosis of our patient. A few months later, a doctor of our team participated in a course for rare diseases, organized by neurologists, where a disease called transthyretin amyloidosis, presenting with neurological, cardiac and GI symptoms, was discussed. This prompted us to refer our patient to a Centre for Rare Neuro-Muscular Diseases, where the neurological investigation showed a sensorimotor polyneuropathy, areflexia in the lower limbs, hyporeflexia in the upper limbs, and the electroneuronography showed peripheral lesion of both sensory and motor fibers of the examined nerves.

The patient was further referred for DNA analysis, which detected a Glu89Gln mutation in exon 3 of the TTR gene and the final diagnosis of ATTR was made. Treatment with the orphan drug tafamidis [7] was started and the patient currently shows no disease progression.

\section{DISCUSSION}

Clinical symptoms of ATTR vary depending on the genetic mutation and on the involved organs, and include a variety of neurological, cardiac, ophthalmic and GI manifestations.

Gastrointestinal manifestations play a key role in patients morbidity, mortality and quality of life $[8,9]$. In some cases, GI symptoms may be present even before the onset of peripheral polyneuropathy. Diarrhea could be the first symptom of this disease [10] and in later stages it becomes prolonged and accompanied by faecal incontinence and/or severe malnutrition $[11,12]$. However, the complaints are various and it is difficult to differentiate them from other GI disorders. The pathogenesis of GI manifestations in ATTR is not fully elucidated; however, motility disturbances due to autonomic nerves denervation are suggested [13].

According to a large international study of GI manifestations in ATTR patients, unexplained weight loss is the most commonly reported GI symptom of the disease [13]. However, this symptom is multifactorial and significant weight loss was observed in patients with ATTR even before the onset of any other symptoms $[13,14]$. That is exactly what happened to our patient. Digestive symptoms are more common in ATTR patients than in the general population. They are more common in the early onset of the disease and increase with the duration of the disease and are associated with a decrease of the healthrelated quality of life [13].

Transthyretin amyloidosis diagnosis is a real challenge for the modern clinician, especially the gastroenterologist, due to the relatively rare GI onset of the disease and the broad differential diagnosis. The disease can easily be misdiagnosed with a number of other GI tract diseases. The presence of specific clinical settings could navigate the physician to the correct diagnosis and help him decide in whom to perform a genetic test: presence of diarrhea and cardiomyopathy and/or polyneuropathy; unintentional weight loss in a patient with neuropathy and/or cardiomyopathy, and a familial history of ATTR.

Essential for the correct diagnosis of ATTR is the organization of multidisciplinary meetings, which by raising the awareness among the physicians could help them include this disorder in their diagnostic work-up. It was such a meeting that helped us to diagnose our patient and to start treatment.

Interestingly, the rectal biopsies with Congo Red staining did not detect amyloid inclusions in our case. The reasons for this could be: the variable rectal biopsy sensitivity, the different staining protocols for Congo Red, the pathologist's experience, and the different sensitivity and specificity of the 
antibodies used for standard immunohistochemistry [5]. Therefore, it is very important that an experienced pathologist evaluates the amyloid deposits, thus reducing the risk of false negative results $[5,15]$. Moreover, during the second visit of the patient, we performed only a rectoscopy with rectal biopsies, because he refused bowel preparation. Recently, a retrospective observational study on amyloid in biopsies of the GI tract found that ATTR amyloid is most prevalent in the rectum (20.5\%), followed by colon (20.1\%), jejunum/ileum (10.3\%), stomach (9.8\%) and duodenum (9.5\%) [16]. Hence, in some cases with ATTR amyloidosis, histology might not be informative.

\section{CONCLUSION}

ATTR is frequently misdiagnosed with many other GI diseases and leads to diagnostic challenges in GI practice. The presence of certain clinical combinations could help gastroenterologists to include ATTR in their diagnostic workup.

Conflicts of interest: None to declare.

Authors' contribution: R.N. and V.N. collected the data and wrote the manuscript. S.S., M.G., T.T., A.K. and A.T. were responsible for patient diagnosis, analysis and treatment. I.T. supervised the whole team. All the authors approved the final version of the manuscript.

\section{REFERENCES}

1. Planté-Bordeneuve V, Said G. Familial amyloid polyneuropathy. Lancet Neurol 2011;10:1086-1097. doi:10.1016/S1474-4422(11)70246-0

2. Olsson M, Hellman U, Planté-Bordeneuve V, Jonasson J, Lång K, Suhr OB. Mitochondrial haplogroup is associated with the phenotype of familialamyloidosis with polyneuropathy in Swedish and French patients. Clin Genet 2009;75:163-168. doi:10.1111/j.13990004.2008.01097.x

3. Ihse E, Ybo A, Suhr O, Lindqvist P, Backman C, Westermark P. Amyloid fibrilcomposition is related to the phenotype of hereditary transthyretin V30M amyloidosis. J Pathol 2008;216:253-261. doi:10.1002/path.2411

4. Quintas A, Vaz DC, Cardoso I, Saraiva MJ, Brito RM. Tetramer dissociationand monomer partial unfolding precedes protofibril formation inamyloidogenic transthyretin variants. J Biol Chem 2001;276:27207-27213. doi:10.1074/jbc.M101024200
5. Adams D, Suhr OB, Hund E, et al. First European consensus for diagnosis,management, and treatment of transthyretin familial amyloid polyneuropathy. Curr Opin Neurol 2016;29 Suppl 1:S14-S26. doi:10.1097/WCO.0000000000000289

6. Volta U, De Giorgio R, Caio G, Uhde M, Manfredini R, Alaedini A. Nonceliac wheat sensitivity: an immune-mediated condition with systemic manifestations. Gastroenterol Clin North Am 2019;48:165182. doi:10.1016/j.gtc.2018.09.012

7. Lorenzini M, Elliott PM. Tafamidis for the treatment of transthyretin amyloidosis. Future Cardiol 2019;15:53-61. doi:10.2217/fca-2018-0078

8. Suhr O, Danielsson A, Holmgren G, Steen L. Malnutrition and gastrointestinal dysfunction as prognostic factors for survival in familial amyloidotic polyneuropathy. J Intern Med 1994;235:479-485. doi:10.1111/j.1365-2796.1994.tb01106.x

9. Suhr O, Danielsson A, Rydh A, Nyhlin N, Hietala SO, Steen L. Impact of gastrointestinal dysfunction on survival after liver transplantation for familial amyloidotic polyneuropathy. Dig Dis Sci 1996;41:1909-1914. doi:10.1007/BF02093589

10. Obayashi K, Olsson M, Anan I, et al. Impact of serotonin transporter andcatechol-O-methyl transferase genes polymorphism on gastrointestinaldysfunction in Swedish and Japanese familial amyloidotic polyneuropathypatients. Clin Chim Acta 2008;398:10-14 doi:10.1016/j.cca.2008.07.033

11. Suhr OB, Svendsen IH, Andersson R, Danielsson A, Holmgren G, Ranlov PJ. Hereditary transthyretin amyloidosis from a Scandinavian perspective. J Intern Med 2003;254:225-235. doi:10.1046/j.13652796.2003.01173.x

12. Conceição I. Clinical features of TTR-FAP in Portugal. Amyloid 2012;19 Suppl 1:71-72. doi:10.3109/13506129.2012.673184

13. Wixner J, Mundayat R, Karayal ON, et al; THAOS investigators. THAOS: Gastrointestinal manifestations of transthyretin amyloidosis common complications of a rare disease. Orphanet J Rare Dis 2014;9:61. doi:10.1186/1750-1172-9-61

14. Wixner J, Karling P, Rydh A, et al. Gastric emptying in hereditary transthyretin amyloidosis: the impact of autonomic neuropathy. Neurogastroenterol Motil 2012;24:1111-e568. doi:10.1111/j.13652982.2012.01991.x

15. Picken MM, Westermark P. Amyloid detection and typing: summary of current practice and recommendations of the consensus group. Amyloid 2011;18 Suppl 1:48-50. doi:10.3109/13506129.2011.574354017

16. Freudenthaler S, Hegenbart U, Schönland S, Behrens HM, Krüger S, Röcken C. Amyloid in biopsies of the gastrointestinal tract-a retrospective observational study on 542 patients. Virchows Arch 2016;468:569-577. doi:10.1007/s00428-016-1916-y 